\title{
A Consideration of Constitutive Relationship between Flowing Particles ${ }^{\dagger}$
}

\author{
Junya Kano \\ Institute for Advanced Materials Processing, Tohoku University* \\ Atsuko Shimosaka and Jusuke Hidaka \\ Department of Chemical Engineering and Materials Science, \\ Faculty of Engineering, Doshisha University**
}

\begin{abstract}
In order to analyze the flow behavior of particles by the application of continum mechanics, a new constitutive equation is derived from considerations of energy dissipation during the flow of the particles. This dissipation mechanism was investigated by computer simulation of the flow behavior of particles on an inclined trough. The energy of flowing particles is dissipated by friction and collision with neighboring particles. The constitutive equation was derived by considering both dissipation mechanisms of energy. The velocity distribution of flowing particles on the inclined trough was estimated by the application of a constitutive equation. The estimated velocity distribution agrees well with the measured one.
\end{abstract}

\section{Introduction}

Computer simulations now provide very useful information on powder systems, and they have become a highly important tool in the study of powder phenomena. In particular the particle element method' provides very detailed spatial and temporal information on the flow behavior of parti. cles, and it has demonstrated its great usefulness in the essential understanding and mechanical analysis of powder behavior ${ }^{2}$. However, as this method entails tracking the movements of individual particles in Lagrangian method, there is a severe limitation on the number of particles in the system being studied, which means it is impossible to simulate particle behavior inside an apparatus working with a very large number of particles. This is a serious problem for the particle element method, which has accomplished much.

Further advances in particle simulations will therefore necessitate the devising of a new simulation method that is unrestricted by the number of particles. One requirement for this new simulation method is quite clear : It should basically be grounded in continuum mechanics, and have a high degree

* 2.1-1, Katahira, Aoba-ku, Sendai 980-77, Japan

**1-3, Tataramiyakodani, kyotanabe, kyoto 610-03, Japan

- This report was originally printed in J. Soc. Powder Technology, Japan. 33, 95 (1996) in Japanese, before being translated into English by KONA Editiorial Committee with the permission of the editiorial committee of the Soc. Powder Technology, Japan. of freedom in element movement in terms of expressing the discrete character of powder behavior. Achieving this kind of simulation method requires, more than anything else, the ability to suitably express powder flows with continuum mechanics.

In this connection, people have for many years assiduously pursued research using polar fluid theory grounded in Cosserat's continuum theory ${ }^{3}$. Kanatani $i^{4}$ in particular developed a theory of pow. der flow on the basis of polar fluid theory. In his work he derived a constitutive relationship for powder flows from macroscopic considerations that assume a particle bed is a continuum, and from microscopic considerations on the state of movement of constitutive particles. The constitutive equations derived are highly useful because they take into consideration the rotation of constitutive particles, which is a characteristic of particle flows, and because there are few parameters that must be obtained experimentally.

But as there was no means of obtaining detailed information on the flow state of a particle bed, there was no consideration of the energy dissipation occurring in conjunction with particle collisions, which have a large effect on particle flow.

As detailed in this report, we used the results ${ }^{\bar{D}}$ from a particle element method simulation of a flow in an inclined trough, which is a comparatively simple particle bed flow, to perform a detailed examination of energy dissipation in a flowing 
Pparticle bed, and derive a constitutive relationship that takes into consideration energy dissipation caused not only by inter-particle friction, but also by particle collisions. We then applied this constitutive relationship to analyze the flow state of a particle bed in an inclined trough and verified its soundness.

\section{Controlling Equations of a Flowing Particle Bed}

\subsection{Fundamental Equations}

Let us assume that a particle bed with a particle size distribution flows in a comparatively dense state, and that inertial force controls its flow. Further, we'll assume that the particles in a flowing bed are always rotating independently instead of submitting to the flow. This being so, the equation of continuity, equation of motion, and equation of angular momentum are as follows.

$$
\begin{gathered}
\frac{\mathrm{d} \rho}{\mathrm{d} t}+\rho \partial_{k} v_{k}=0 \\
\rho \frac{\mathrm{d} v^{i}}{\mathrm{~d} t}=f_{i}+\partial_{j} \sigma^{j i} \\
\rho \frac{\mathrm{d} \omega_{j i}}{\mathrm{~d} t}=\frac{10}{D_{s}{ }^{2}}\left\{2 \sigma^{[j i]}+\partial_{k} \mu^{k j i}\right\}
\end{gathered}
$$

Where :

$v$ is particle velocity,

$\rho$ is bulk density,

$\sigma$ is stress,

$f$ is external force,

$\omega$ is rotational velocity,

$\mu$ is couple stress, and

$D_{s}$ is particle diameter of mean surface.

\subsection{Energy Dissipation in a Flowing Particle Bed}

Generally constitutive equations are determined by two controlling functions: free energy and a dissipation function. We'll assume that in the flow of a particle bed the collective has no internal energy, all internal force being dissipation energy. On the basis of this assumption, Kanatani focussed on the energy dissipation of particle mass, and derived a constitutive equation by determining the equivalent stress which causes that dissipative work.

This method requires detailed information on energy dissipation. The authors previously used the particle element method, which provides detailed information on the behavior of flowing particle beds, to simulate a particle bed flowing in an inclined trough ${ }^{5}$. Figs. 1 and 2 show the initial particle fill in a trough, and the particle velocity distribution at the side of the inclined trough, respectively. Table 1 shows the calculation conditions and physical constants used in the simulation. Figs. $3(\mathbf{a})$ and (b) show the dissipated energy by friction $\Phi_{f}$ and dissipated energy by collision $\Phi_{c}$ obtained from simula tion results. Table 2 shows the ratio $R$ of energy dissipated by collisions to total dissipated energy. As the trough's angle of inclination becomes greater, the dissipated energy caused by both fric-

Table 1 Physical constants used in simulation calculation

\begin{tabular}{|l|c|c|}
\hline Young's modulus & $4.9 \times 10^{9}$ & {$[\mathrm{~Pa}]$} \\
(wall) & $3.9 \times 10^{9}$ & {$[\mathrm{~Pa}]$} \\
Poisson's ratio & 0.23 & {$[-]$} \\
(wall) & 0.25 & {$[-]$} \\
Number of particles & 3752 & {$[-]$} \\
Particle diameter & 20 & {$[\mathrm{~mm}]$} \\
Time step & $1.0 \times 10^{-5}$ & {$[\mathrm{~s}]$} \\
Coefficient of repulsion & 0.8 & {$[-]$} \\
Coeffisient of friction & 0.466 & {$[-]$} \\
(particle-wall) & 0.268 & {$[-]$} \\
Particle dnsity & $2.65 \times 10^{3}$ & {$\left[\mathrm{~kg} / \mathrm{m}^{3}\right]$} \\
\hline
\end{tabular}

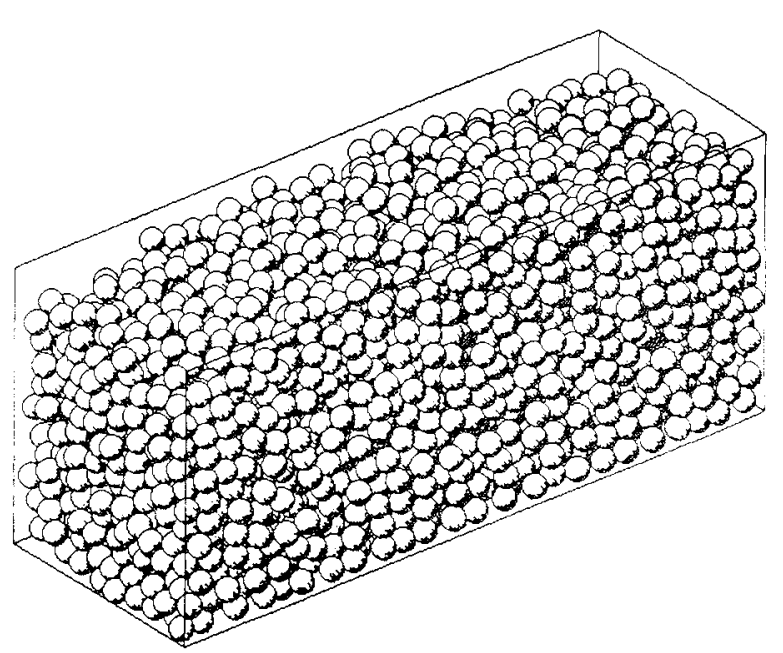

Fig. 1 Initial fill of particles in trough

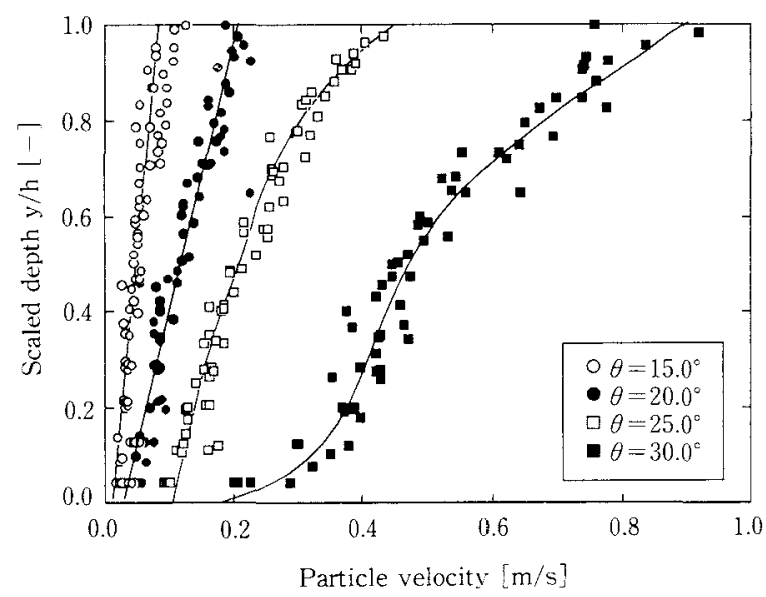

Fig. 2 Velocity profiles obtained in 3D simulation calculation of chute flow 
Table 2 Mean dissipation energy of flowing particles and ratio of energy dissipated by collisions to total dissipation energy

\begin{tabular}{|c|c|c|c|}
\hline$\theta\left[{ }^{\circ}\right]$ & $E_{f}[\mathrm{~J}]$ & $E_{c}[\mathrm{~J}]$ & $R[-]$ \\
\hline 10.0 & 0.134 & $2.00 \times 10^{-2}$ & 0.129 \\
15.0 & 0.252 & $3.90 \times 10^{-2}$ & 0.134 \\
20.0 & 0.482 & $9.36 \times 10^{-2}$ & 0.163 \\
25.0 & 0.983 & 0.257 & 0.207 \\
30.0 & 1.663 & 0.921 & 0.356 \\
\hline
\end{tabular}
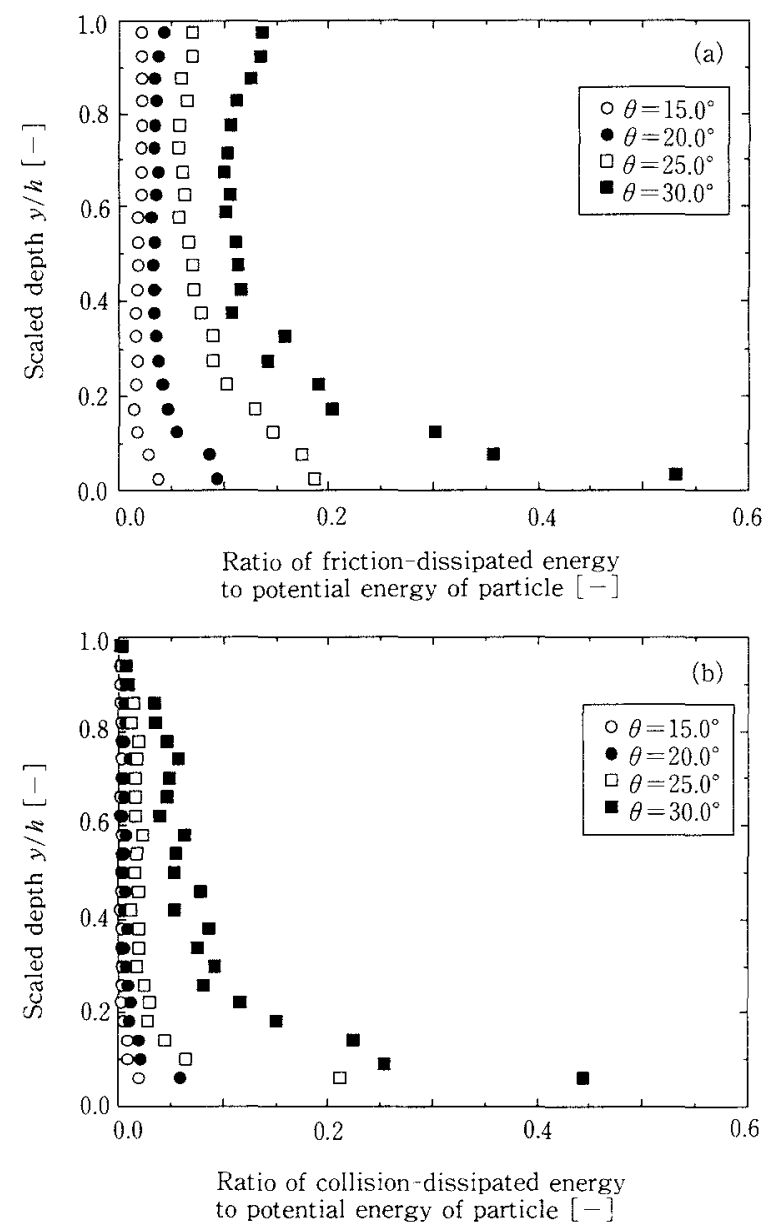

Fig. 3 Dissipation energy of flowing particles on chute

tion and particle collisions becomes greater, and the ratio of collision-caused dissipated energy to total dissipated energy grows. Especially when chute flow becomes large, the ratio of collision-caused dissipated energy to total dissipated energy attains 30 or $40 \%$, a value that cannot be ignored. Thus, in the derivation of a constitutive equation applied to particle bed flows, one must take into consideration collision-caused dissipated energy in addition to friction-caused dissipated energy.

\subsection{Constitutive Equation for a Flowing Particle Bed}

The total dissipated energy $\Phi_{m}$ of a flowing particle bed is the sum of dissipated energy by friction $\Phi_{f}$ and dissipated energy by collision $\Phi_{c}$, as shown in this equation.

$$
\Phi_{m}=\Phi_{f}+\Phi_{c}
$$

Next these values for dissipated energy are deter mined by using a model of the flowing particle bed. In particle flows with comparatively little mutual inter-particle action and high fluidity, individual particles flow while continuously colliding with nearby particles. This flowing particle movement can be demonstrated using the cell model ${ }^{4}$ in Fig. 4. It shows that a certain particle with microscopic velocity $v^{\prime}$ moves randomly in a spherical space with diameter $D_{r}$, and that it flows while continuously colliding with the cell wall (i.e., nearby particles in the first stratum). We have already examined in detail the applicability of the cell model to particle bed flows using the results of a particle element method simulation of a particle bed flowing in an inclined trough ${ }^{5}$. On the basis of this cell model it is possible to determine the flowing particle bed's friction- and collision-caused dissipated energy.

\section{a) Energy Dissipated by Friction}

When a certain particle collides with its cell wall, the compressive force acting on the wall is $\pi D_{r}^{2} P$ when average pressure is $P$, and frictional force is $\mu_{i} \pi \mathrm{D}_{r}^{2} P$ when $\mu_{i}$ is the frictional coefficient. If the tangential component of collision velocity at the point of collision is $\xi_{\text {i }}$, the energy dissipated by friction that is caused by the collision is expressed as $\mu_{i} \pi D_{r}^{2} P \xi_{i}$. Thus the energy dissipated by friction

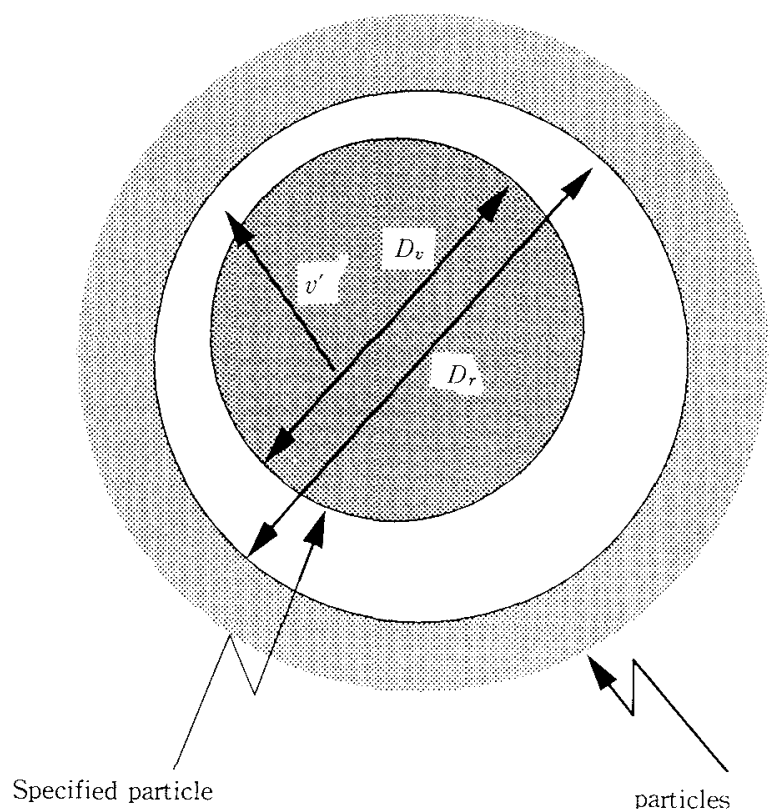

Fig. 4 Cell model of flowing particles 
Pperwathit volume and unit time of the flowing particles is determined by the following equation.

$$
\Phi_{f}=\frac{\pi}{2 m} \mu_{i} D_{r}^{2} \rho P \xi_{i}
$$

Because the particle continuously collides with its spherical cell wall, the mean pressure $P$ in Equation (5) can be found in the same way as in the kinetic theory of gases. In other words, the distance that the particle can move without colliding with the wall of its spherical cell, whose diameter is $D_{r}$, is $\left(D_{r}-D_{v}\right) \sin \zeta$ when the collision angle is $\zeta$. Thus, the number of collisions $n$ per unit time is $2 v^{\prime} e /(1+$ e) $\times\left(D_{r}-D_{v}\right) \sin \zeta$, using $v^{\prime}$ for particle microscopic velocity and e for the coefficient of repulsion. Each collision transmits a momentum of $m v^{\prime}(1+e) \sin \zeta$ to the cell wall, meaning that a force of $n m v^{\prime}(1+e)$ $\sin \zeta$ acts on the wall per unit time. As cell surface area is $\pi D_{r}{ }^{2}$, mean pressure $\mathrm{P}$ is found by the following equation.

$$
P=\frac{2 m v^{\prime 2} e}{\pi D_{r}^{2}\left(D_{r}-D_{v}\right)}, m=\frac{\pi}{6} D_{v}^{3} \rho_{p}, D_{r}=D_{v} \sqrt[3]{\frac{\rho_{0}}{\rho}}
$$

Where :

$\rho_{0}$ is the bulk density of the initial particle fill.

Thus, if Equation (6) is substituted with Equation (5), energy dissipated by friction is found by this equation.

$$
\Phi_{f}=\frac{\mu_{i} e v^{\prime 2} \rho \xi_{i}}{D_{r}-D_{v}}
$$

\section{b) Energy Dissipated by Collisions}

Because the energy dissipated by one collision is $(1 / 2) m v^{\prime 2} \times(1-e)^{2}$, the energy per unit time is (1/ 2) $n m v^{\prime 2} \times(1-e)^{2}$. Thus the dissipated energy according to collisions per unit volume is determined by this equation.

$$
\Phi_{c}=\frac{1}{2} \frac{\rho v^{\prime 3}}{D_{r}-D_{v}} \frac{e(1-e)^{2}}{(1+e) \sin \zeta}
$$

If Equation (4) is substituted for Equations (7) and (8), total dissipated energy of a flowing particle bed is determined in the following manner.

$$
\Phi_{m}=\frac{\mu_{i} e v^{\prime 2} \rho \xi_{i}}{D_{r}-D_{v}}+\frac{1}{2} \frac{v^{\prime 3} \rho}{D_{r}-D_{v}} \frac{e(1-e)^{2}}{(1+e) \sin \zeta}
$$

The following equations determine the tangential component $\zeta_{i}$ of the relative velocity in collisions between the particle and its cell wall ${ }^{4}$.

$$
\begin{gathered}
\hat{\xi}_{i}=\frac{\sqrt{6}}{3} D_{s} \hat{\omega}, \hat{\omega}= \\
\sqrt{\frac{3}{10} E_{j i} E_{j i}+\frac{1}{2} R_{j i} R_{j i}+\frac{D_{s}^{2}}{40}\left(\Omega_{k k j} \Omega_{l i j}+\Omega_{k j i} \Omega_{k j i}+\Omega_{k j i} \Omega_{k i j}\right)} \\
E_{j i}=D_{(j i)}-\frac{1}{3} \delta_{j i} D_{k k}, D_{j i}=\partial_{j} v_{i}, \\
R_{j i}=\omega_{j i}-D_{[j i}, \Omega_{k j i}=\partial_{k} \omega_{j i}
\end{gathered}
$$

Substituting Equation (9) for Equation (10), total dissipated energy is determined by the following equation.

$$
\begin{gathered}
\Phi_{m}=C(\rho) \hat{\omega}^{3} \\
C(\rho)=\frac{\sqrt{6}}{30} \cdot \frac{\mu_{i} e T D_{s}^{3} \rho}{D_{r}-D_{v}}+\frac{\sqrt{10}}{200} \frac{T \sqrt{T} D_{s}^{3} \rho}{D_{r}-D_{v}} \frac{e(1-e)^{2}}{(1+e) \sin \zeta}
\end{gathered}
$$

Where :

$T$ is the ratio of the particle's translational kinetic energy $\left(1 / 2 m v^{\prime 2}\right)$ to rotational kinetic energy.

Macroscopic dissipated energy $\Phi_{s}$, however, can be obtained from the law of energy conservation. Assuming that power by external force is equal to the increase in kinetic energy and the dissipated energy per unit time, the following equation determines macroscopic dissipated energy ${ }^{4}$.

$$
\begin{gathered}
\Phi_{s}=\tilde{\sigma}^{j i} E_{j i}-\sigma^{[j i]} \omega_{j i}+\frac{1}{2} \mu^{k j i} \Omega_{k j i} \\
\bar{\sigma}^{j i}=\sigma^{j i}-\frac{1}{3} \delta_{i j} \sigma^{k k}
\end{gathered}
$$

Combining the microscopic dissipated energy found by the cell model (Equation (9)) with the macroscopic energy determined by the law of energy conservation yields the following constitutive equations ${ }^{4}$.

$$
\begin{gathered}
\sigma^{j i}=C(\rho) \hat{\omega}\left(\frac{2}{5} \frac{\partial v_{j}}{\partial x_{i}}-\frac{1}{10} \frac{\partial v_{i}}{\partial x_{j}}+\frac{1}{2} \omega_{j i}\right) \\
\sigma^{i i}=\frac{1}{5} C(\rho) \hat{\omega} \frac{\partial v_{i}}{\partial x_{i}}-P \\
\sigma^{[j i]}=\frac{1}{2} C(\rho) \hat{\omega}\left(\frac{1}{2} \frac{\partial v_{j}}{\partial x_{i}}-\frac{1}{2} \frac{\partial v_{i}}{\partial x_{j}}+\omega_{j i}\right) \\
\mu^{k j i}=\frac{D_{s}^{2}}{40} C(\rho) \hat{\omega} \frac{\partial \omega_{j i}}{\partial x_{k}}
\end{gathered}
$$

Now we shall use the fundamental equations in section 2.1 and the constitutive equations obtained here to attempt an analysis of a typical particle flow state.

\subsection{Numerical Analysis of Particles Flowing Through an Inclined Trough}

We conceived a flow such as that in Fig. 5 in order to compare the constitutive equations proposed here with the analysis results given by Kanatani's equations, which do not take into consideration the energy dissipation caused by collision. Following are the equations of motion for this particle flow.

$$
\begin{gathered}
\rho \frac{\mathrm{d} u}{\mathrm{~d} t}=C(\rho) \frac{\partial}{\partial y}\left\{\omega\left(\frac{2}{5} \frac{\partial u}{\partial y}+\frac{1}{2} \omega\right)\right\}+\rho g \sin \theta \\
\rho \frac{\mathrm{d} \omega}{\mathrm{d} t}=\frac{10 C(\rho)}{D_{s}^{2}}\left\{\hat{\omega}\left(\frac{1}{2} \frac{\partial u}{\partial y}+\omega\right)+\frac{D_{s}^{2}}{40} \frac{\partial}{\partial y}\left(\hat{\omega} \frac{\omega}{\partial y}\right)\right\} \\
\bar{\omega}=\sqrt{\frac{1}{2}\left(\frac{1}{2} \frac{\partial u}{\partial y}+\omega\right)^{2}+\frac{3}{40}\left(\frac{\partial u}{\partial y}\right)^{2}+\frac{D_{s}^{2}}{10}\left(\frac{\partial \omega}{\partial y}\right)^{2}}
\end{gathered}
$$

By contrast, Kanatani's equations for the same 
Table 3 Physical constants used in FDM analysis

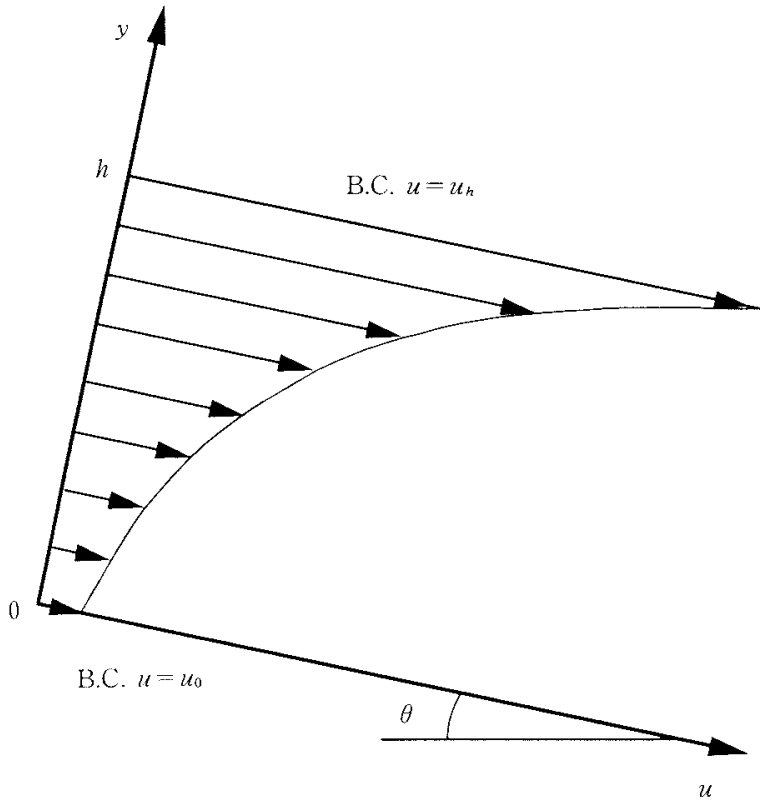

Fig. 5 Boundary condition for calculation of velocity distribu tion of flowing particles on chute

flow are as follows.

$$
\begin{gathered}
\rho \frac{\mathrm{d} u}{\mathrm{~d} t}=B(\rho) \frac{\partial}{\partial y}\left\{\omega\left(\frac{2}{5} \frac{\partial u}{\partial y}+\frac{1}{2} \omega\right)\right\}+\rho g \sin \theta \\
\rho \frac{\mathrm{d} \omega}{\mathrm{d} t}=\frac{10 B(\rho)}{D_{s}^{2}}\left\{\omega\left(\frac{1}{2} \frac{\partial u}{\partial y}+\omega\right)+\frac{D_{s}^{2}}{40} \frac{\partial}{\partial y}\left(\hat{\omega} \frac{\partial \omega}{\partial y}\right)\right\} \\
B(\rho)=\frac{\sqrt{6} \pi}{60} \frac{T \mu_{i} D_{r}{ }^{2} D_{p}{ }^{3}}{m} \frac{\rho_{0} \rho^{2}}{\rho_{0}-\rho}
\end{gathered}
$$

Where :

$D_{p}$ is particle size.

For each equation of motion, one approximates by forward difference for time and central difference for space, and obtains numerical solutions using the finite difference method. By way of boundary conditions, for particle velocities $u_{0}$ and $u_{h}$ at the lowest and highest lattice points we assigned the particle velocity values observed for the bottom and top of the particle bed in a flow experiment with the inclined trough (Fig. 6) ${ }^{5}$. For initial conditions we used the particle velocities yielded by the following equation.

$$
u=\left(u_{h}-u_{0}\right) y / h+u_{0}
$$

In Table 3 are the calculation conditions and physical constants used in these calculations. We assumed a constant bulk density for the state of particle flow, and made the ratio $T$ of the particle' $s$ translational energy to rotational energy match the observed values for particles flowing through the trough.

\section{Results and Discussion}

Figs. $7(\mathbf{a})$ and (b) show the particle velocity

\begin{tabular}{|l|c|c|}
\hline$\Delta t$ & $1.0 \times 10^{-5}$ & {$[\mathrm{~s}]$} \\
$\Delta y$ & $5.0 \times 10^{-4}$ & {$[\mathrm{~m}]$} \\
$e$ & 0.78 & {$[-]$} \\
$\mu_{i}$ & 0.380 & {$[-]$} \\
$D_{s}$ & 2.0 & {$[\mathrm{~mm}]$} \\
$D_{v}$ & 2.0 & {$[\mathrm{~mm}]$} \\
$D_{p}$ & 2.0 & {$[\mathrm{~mm}]$} \\
$\rho_{0}$ & $1.563 \times 10^{3}$ & {$\left[\mathrm{~kg} / \mathrm{m}^{3}\right]$} \\
$\rho$ & $1.548 \times 10^{3}$ & {$\left[\mathrm{~kg} / \mathrm{m}^{3}\right]$} \\
$h$ & $4.0 \times 10^{-2}$ & {$[\mathrm{~m}]$} \\
\hline
\end{tabular}

distributions obtained by numerical calculations using the same conditions as in the flow experiment that yielded the particle flow distribution in Fig. 6 . Fig. 7 (a) shows the results of Equations (21) and (22) proposed by Kanatani, while Fig. 7 (b) presents the results of Equations (18) and (19), which are proposed in this report. Estimated velocities according to the constitutive equations proposed here are in all cases smaller than those based on Kanatani's constitutive relationship; even in a low particle velocity region when the trough's incline is low, there is a slight difference in the velocity distributions. When the angle of incline $\theta$ is $15.5^{\circ}$ and $16.9^{\circ}$, i.e., inclinations at which particle velocity increases, the difference between the distributions grows. Furthermore, overall the particle velocity distributions estimated using the constitutive equations proposed here more closely match measured values than Kanatani's results.

Figs. 8(a), (b), and (c) illustrate the effects of the coefficient of friction $\mu_{i}$ on particle velocity distribution. A decrease in the frictional coefficient makes for an increase in the difference between the particle velocity obtained under Kanatani's theory and that obtained under the theory proposed here,

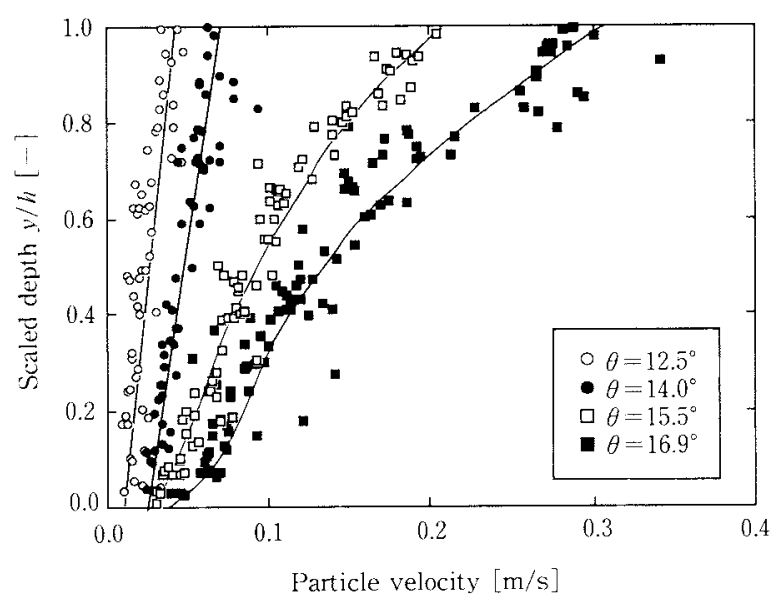

Fig. 6 Velocity profiles obtained in chute flow test 

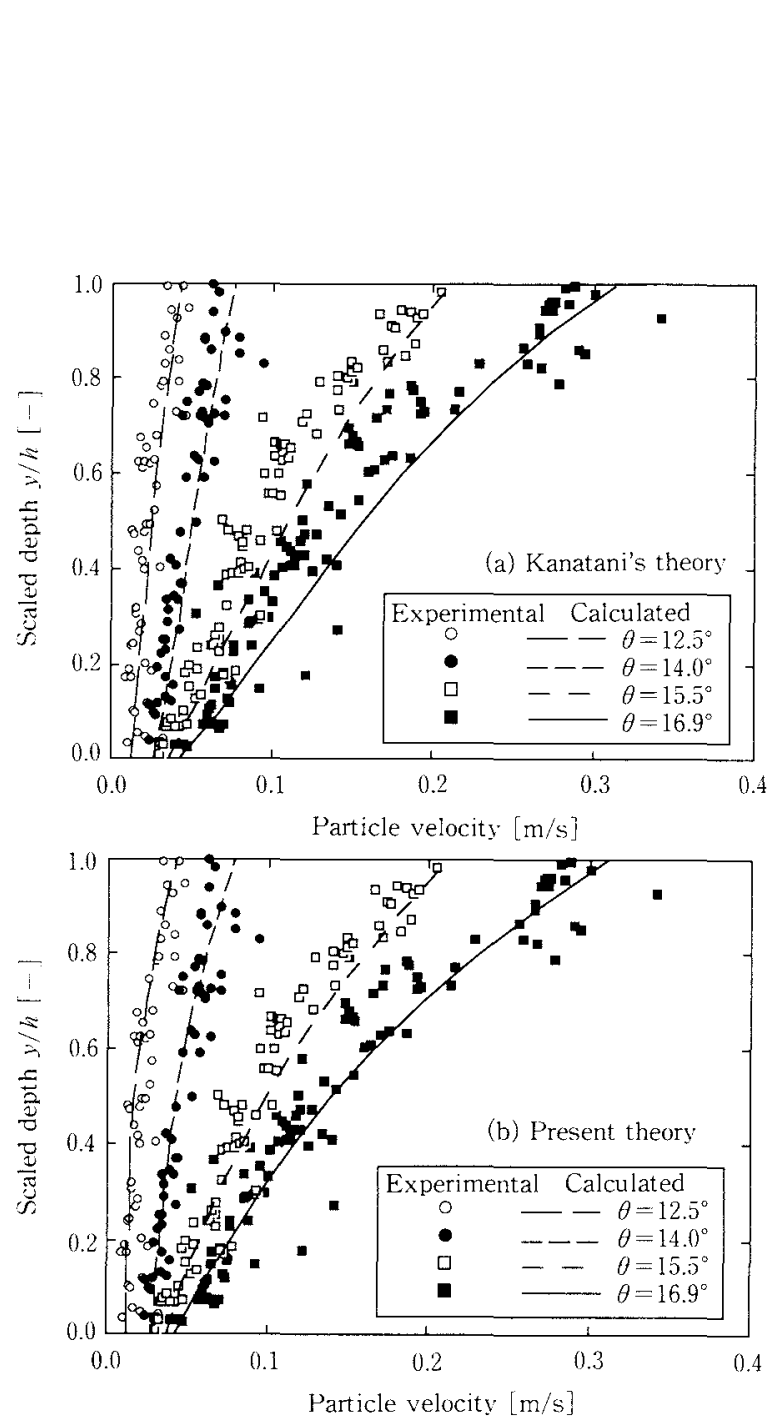

Fig. 7 Velocity profiles estimated by numerical analysis using proposed constitutive equation

signifying an increase in the effect of energy dissipation by collisions.

Fig. 9 shows the ratio of collision-caused dissipated energy to the total dissipated energy derived from numerical calculation results. As the interparticle frictional coefficient and the inter-particle coefficient of repulsion become smaller, it becomes impossible to ignore energy dissipated by collisions.

The foregoing results show that when an ordinary powder (frictional angle of $20^{\circ}$ to $30^{\circ}$ ) flows at a comparatively high velocity, the energy dissipated by collisions has a large effect on powder flow; and that the constitutive equations proposed here are useful over a broad range, from slow to fast flows.

\section{Conclusion}

The authors performed research on constitutive relationships in flowing powder beds so that it would be possible to examine particle flow behavior in terms of continuum mechanics. Using simulations according to the particle element method to carefully observe flow behavior in a powder bed, we found that it is necessary for the flow model and
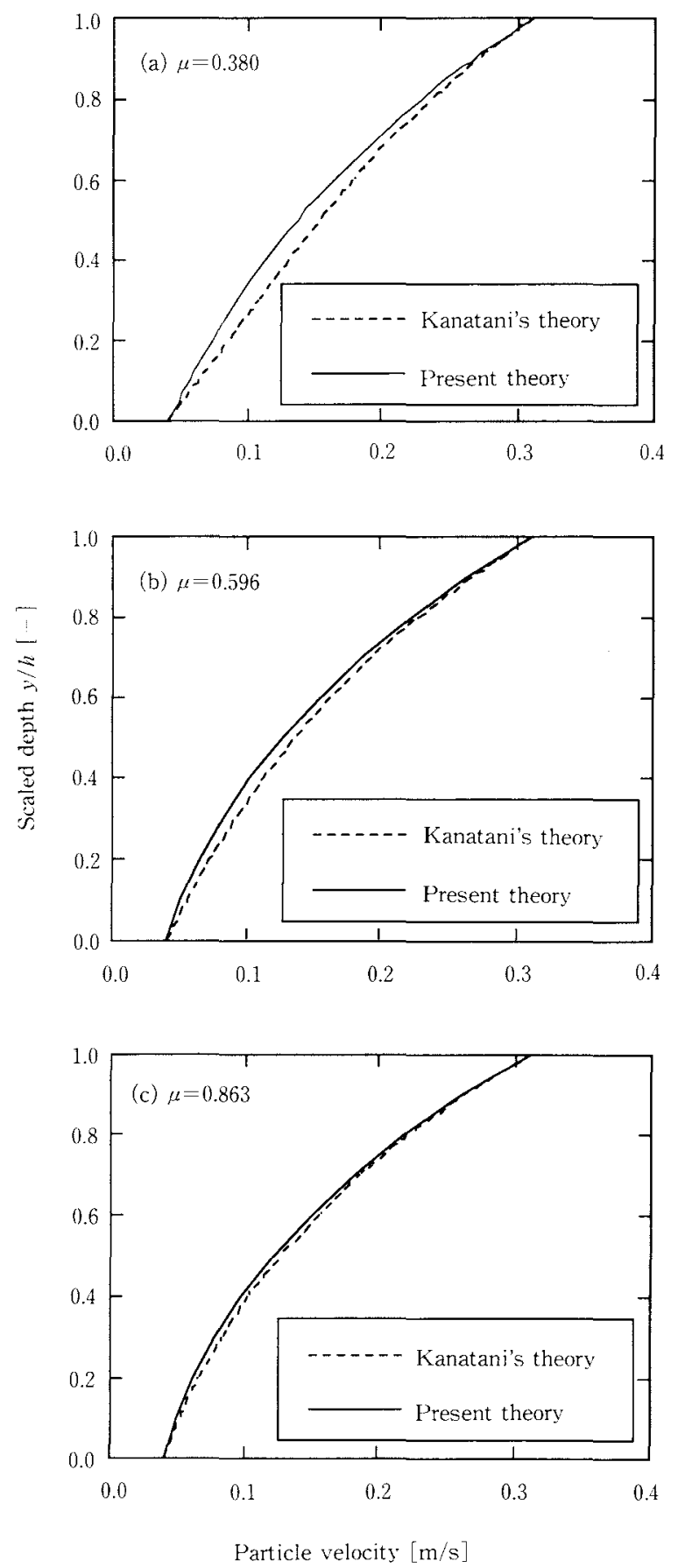

Fig. 8 Effect of coefficient of friction between particles on particle velocity distribution

constitutive equations derived by Kanatani to take collision-caused energy dissipation into account. For this purpose we derived constitutive equations that incorporate the coefficient of repulsion, and used them to obtain velocity distributions for a flowing particle bed in an inclined trough by means of the finite difference method. Calculated velocity distributions closely matched the plots of measured distributions, demonstrating the soundness of incorporating the coefficient of repulsion and modeling it. As the powder flow velocity in powder devices is 


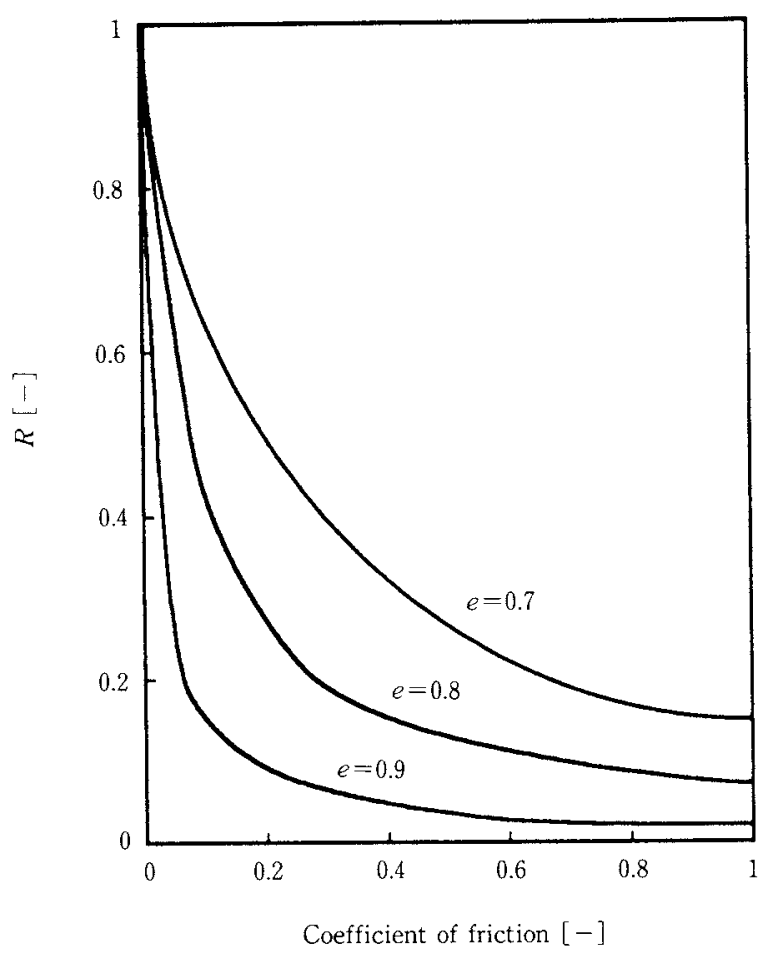

Fig. 9 Effect of coefficient of friction on the ratio of dissipation energy with collision to total dissipation energy

quite fast, we believe that in performing analyses of actual powder behavior it will prove useful to employ the proposed constitutive relationship, which takes collision-caused energy dissipation into account.

\section{Nomenclature}

$B \quad$ : function of $\rho$ defined by Eq. $(23) \quad(\mathrm{kg} / \mathrm{m})$

$C \quad$ : coefficient in Eq. (12) $\quad(\mathrm{kg} / \mathrm{m})$

$D_{j i} \quad$ : tensor of velocty gradient defined by Eq. (11)

$D_{(j i)} \quad: \frac{1}{2}\left(\partial_{j} v^{i}+\partial_{i} v^{j}\right)$

$D_{[j i]} \quad: \frac{1}{2}\left(\partial_{j} v^{i}-\partial_{i} v^{j}\right)$

$D_{p} \quad$ : particle diameter

$D_{r} \quad$ : diameter of cell

$D_{s} \quad$ : particle diameter of mean surface

$D_{v} \quad$ : particle diameter of mean volume

(m)

$E_{j i} \quad$ : tensor of velocity gradient deviator defined by Eq. (11)

$e \quad$ : coefficient of repulsion between particles
: external force

$(\mathrm{N})$

$\left(\mathrm{m} / \mathrm{s}^{2}\right)$

gravitational acceleration

(m)

depth of powder bed

$(\mathrm{kg})$

mass of particle

$(\mathrm{Pa})$

$P \quad$ : pressure

$R_{j i} \quad$ : relative rotational velocity of particle defined by Eq. (11)

$T$ : ratio of kinetic energy of specified particle to rotational kinetic energy

: time

$u \quad$ : x-component of particle velocity $(\mathrm{m} / \mathrm{s})$

$u_{0}$ : x-component velocity of particle at the bottom of powder bed $(\mathrm{m} / \mathrm{s})$

: $\mathrm{x}$-component velocity of particle at the top of powder bed $(\mathrm{m} / \mathrm{s})$

: particle velocity (m/s)

: speed of specified particle $(\mathrm{m} / \mathrm{s})$

: height of particle in powder bed (m)

: Kronecker's delta ( - )

: inclinational angle of chute (deg)

: couple stress

$(\mathrm{Pa} / \mathrm{m})$

: coefficient of friction (-)

: angle of collision (deg)

: tangential component of relative velocity

$(\mathrm{m} / \mathrm{s})$

: bulk density of flowing particles $\left(\mathrm{kg} / \mathrm{m}^{3}\right)$

$\rho_{0} \quad$ : bulk density at initial packing of particles

$\left(\mathrm{kg} / \mathrm{m}^{3}\right)$

$\rho_{p} \quad:$ density of particle $\quad\left(\mathrm{kg} / \mathrm{m}^{3}\right)$

$\sigma \quad:$ stress $\quad(\mathrm{Pa})$

$\sigma^{(j i)} \quad: \frac{1}{2}\left(\sigma_{j i}+\sigma_{i j}\right)$

$\sigma^{[j i]} \quad: \frac{1}{2}\left(\sigma_{j i}-\sigma_{i j}\right)$

$\Phi_{c} \quad$ : dissipation energy by collision $\quad(\mathrm{J} / \mathrm{s})$

$\Phi_{f} \quad$ : dissipation energy by friction $\quad(\mathrm{J} / \mathrm{s})$

$\Phi_{m} \quad$ : microscopic dissipation energy $\quad(\mathrm{J} / \mathrm{s})$

$\Phi_{s} \quad$ : macroscopic dissipation energy $\quad(\mathrm{J} / \mathrm{s})$

\section{References}

1) Hidaka J.: J. Soc. Powder Technol., Japan, 29, 465 (1992)

2) Hidaka J., J. Kano and A. Shimosaka : Kagaku Kogaku Ronbunshu, 20, 397 (1994)

3) Oshima N. : Zairyo Kagaku, 5, 294 (1972)

4) Kanatani K. : Nihon kikai Gakkai Ronbunshu, 45-392, 507 (1979)

5) Kano J., A. Shimosaka and J. Hidaka: J. Soc. Powder Technol., Japan, 30, 183 (1993) 


\section{Author's short biography}

\section{Junya Kano}

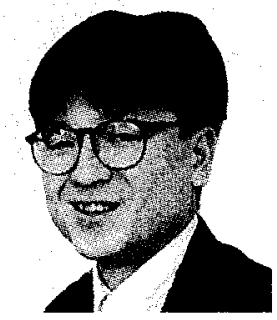

Junya Kano is a research associate at the Institute for Advanced Materials Processing (IAMP), Tohoku University. He graduated in the postgraduate course of department of chemical engineering, Doshisha University in 1995 and took a doctorate in engineering from Doshisha University in 1997. His main fields of research are in the computer simulations of powder flow and balls' behavior in mills and material science of ceramic powder.

\section{Atsuko Shimosaka}
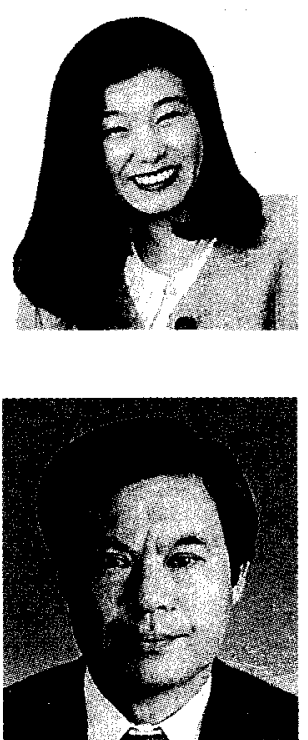

\section{Jusuke Hidaka}

Prof. Jusuke Hidaka has been at Doshisha University since 1976. His major research interests lie in mechanical analysis of powder flow using computer simulation, classification and instrumentation of powder industrial processes using acoustic emission. He is the secretary of the Society of Powder Technology, Japan. 